\title{
Narrow/Broad-Band Absorption Based on Water-Hybrid Metamaterial
}

\author{
Bui Xuan Khuyen ${ }^{1,2, *}$, Vu Thi Hong Hanh ${ }^{3, *}$, Bui Son Tung ${ }^{1,2}$, Vu Dinh Lam ${ }^{1}$, Young Ju Kim ${ }^{4}$, \\ YoungPak Lee ${ }^{5,6, *}$, Hua-Tian Tu ${ }^{6}$ and Liang Yao Chen ${ }^{6}$ \\ 1 Graduate University of Science and Technology, Vietnam Academy of Science and Technology, \\ Hanoi 100000, Vietnam; tungbs@ims.vast.ac.vn (B.S.T.); lamvd@gust-edu.vast.vn (V.D.L.) \\ 2 Institute of Materials Science, Vietnam Academy of Science and Technology, Hanoi 100000, Vietnam \\ 3 Thai Nguyen University of Education, Thai Nguyen University, Thai Nguyen 25000, Vietnam \\ 4 Dongwoo Fine-Chem, Pyeongtaek 17956, Korea; nmkyj@hanmail.net \\ 5 Department of Physics, Quantum Photonic Science Research Center and RINS, Hanyang University, \\ Seoul 04763, Korea \\ 6 Department of Optical Science and Engineering, Fudan University, Shanghai 200433, China; \\ tuhuatian@163.com (H.-T.T.); lychen@fudan.ac.cn (L.Y.C.) \\ * Correspondence: khuyenbx@ims.vast.ac.vn, (B.X.K); hanhvth@tnue.edu.vn, (V.T.H.H.); \\ yplee@hanyang.ac.kr (Y.L.)
}

Received: 5 May 2020; Accepted: 19 May 2020; Published: 22 May 2020

check for updates

\begin{abstract}
In this work, the possibility of a switchable metamaterial absorber is proposed to control absorption bandwidth in the WiMAX/LTE (worldwide interoperability for microwave access/long term evolution) band, by taking advantage of the low cost and myriad structural configurations afforded by water-based metamaterials. By exploiting truncated cone-type resonators, the fractional bandwidth of $27.6 \%$ of absorption spectrum can be adjusted flexibly to be $7.4 \%$ of the narrow-band absorption depending on the volume of injected water, in both simulation and experiment at room temperature. In particular, this control method can be applied stably for different temperature of injected water. We describe a dynamic mechanism for broadband MA, as well as a principle for controlling the absorption characteristics utilizing a combination of magnetic resonance and perfect impedance matching. These results are a stepping-stone towards the realization of smart electronics integrated with multi-functional metamaterials in military, biomedical, communication and other fields.
\end{abstract}

Keywords: metamaterials; perfect absorption; broadband

\section{Introduction}

The idea for metamaterial absorbers (MAs) — which appeared over a decade ago—is that incoming electromagnetic (EM) waves can be trapped inside the sub-wavelength scale, which is not observed in natural materials [1,2]. Effective permeability and permittivity can be controlled flexibly to be equal at the intrinsic resonances by changing the physical structure of assembled materials. Therefore, the operating frequencies of MAs can be tuned from the radio to the visible range, creating extensive applications for both civilian and military uses [3-10]. Multilayer structures have been exploited to achieve broadband absorption and spatial frequency dispersion; however, their bandwidths were hard to tune, owing to the fact that their properties were immutable after fabrication [11-15]. To remedy this, several typical modulatory principles have been intensively developed to achieve multi-functionality, including tunable lumped elements [16], phase-changing materials [17,18] and graphene $[19,20]$. While these approaches offer the ability to excite multiple resonances in close proximity, there are still inherent difficulties due to the fact that multi-absorption peaks are difficult 
to cancel or recover independently. Therefore, it is imperative to develop the improved types of tunable/switchable broadband MAs by hybridization of the modulatory materials in multilayer MAs.

Recently, water has become a good candidate for broadband MAs due to its low conductivity and relatively large imaginary part of the permittivity in the $\mathrm{GHz}$ range (because of the hydrogen-bonded network among water molecules [21]). It is also well known that between 0 and $100^{\circ} \mathrm{C}$ (at normal pressures) water is a liquid that can be easily equipped for mechanical- and thermal-tunable purposes [22-27]. In 2015, Yoo et al. devised that water droplets could be periodically positioned on different substrates to present broadband absorption by exploiting their electric and magnetic resonances [28]. The absorption of the obtained spectra was approximately $93 \%$ from 8 to $18 \mathrm{GHz}$ (for mobile communication, satellite and radar applications). In the higher-frequency bands, $20-40 \mathrm{GHz}$, Song et al. found that a flexible water-based MA achieved near unity electromagnetic absorption by using a water sphere cap sandwiched between top and bottom membranes made of PDMS (polydimethylsiloxane) with a bonded-metallic backside [29]. Their optically transparent absorber could be useful in stealth technology $[30,31]$. To the best of our knowledge, the tunable broadband absorption of water-based MAs in recent literature has mostly concentrated on the frequency range above C-band (over six GHz) where water has high absorption, and the low cost and the ability to make complex structures without masks are advantageous. However, further improvement of broadband absorption at lower frequencies, especially, below six GHz, is an interesting and relatively unexplored challenges. In addition, there is lack of independent-switchable ability for narrow/broad-band MAs, since previous tuning performances were realized totally with effective impedance-matching values (which affect the absorption) by changing any physical feature of water (permittivity, volume, etc.). Moreover, the experimental confirmation was hardly clarified at different temperatures of water, owing to the complicated and/or expensive techniques for those hybrid MAs.

In this work, we propose a simple approach to obtain switchable MAs which can tune their absorption bandwidth to be narrow/broad-band. By hybridization of water into a multilayer MA operating in the WiMAX/LTE (worldwide interoperability for microwave access/long term evolution) band (4-6 GHz), the magnetic resonances can be independently modulated by injection of water to have different volume ratios, which is equivalent to the increase or reduction of each absorption frequency. A proposed water-hybrid MA was tested for the thermo-stable performance, based on both simulation and experiment. We believe that the proposed design can find various applications in manipulating the EM wave-matter interaction.

\section{Materials and Methods}

First, our multilayered sample was designed with CST Microwave Studio to simulate a broadband absorption, as shown in Figure 1a. The unit cells were the truncated cone-type resonators (TCRs) with height $\mathrm{L}$, which utilized 27 sandwiched layers (metal-dielectric-metal) deposited on a substrate with thickness $t_{1}$. The meta-surfaces were circular with diameter varying linearly from $D_{1}$ at the top surface to $\mathrm{D}_{2}$ at the bottom. Dielectric and substrate layers were selected as flame retardant- 4 (FR-4) with a dielectric constant of 4.3 and a loss tangent of 0.03 . The $0.036 \mathrm{~mm}$-thick copper layer (conductivity of $\sigma$ $=5.8 \times 10^{7} \mathrm{~S} / \mathrm{m}$ ) was designed for all metallic layers. The geometrical parameters were optimized to be $D_{2}=21.8, D_{1}=13.8, A=23.8, L=6.3$ and $t_{1}=2.2 \mathrm{~mm}$. As in the enlargement in Figure $1 \mathrm{a}$, a sandwiched layer contained a pair of metallic plates (thickness of $0.036-\mathrm{mm}$ ), which was separated by a dielectric spacer (0.182-mm thick). This lay on a dielectric substrate of $t_{1}=2.2 \mathrm{~mm}$. Second, water was directly injected into the air fissures between adjacent unit cells and its volume was controlled by height $h$. The fabrication procedure is simplified in Figure 1a. 


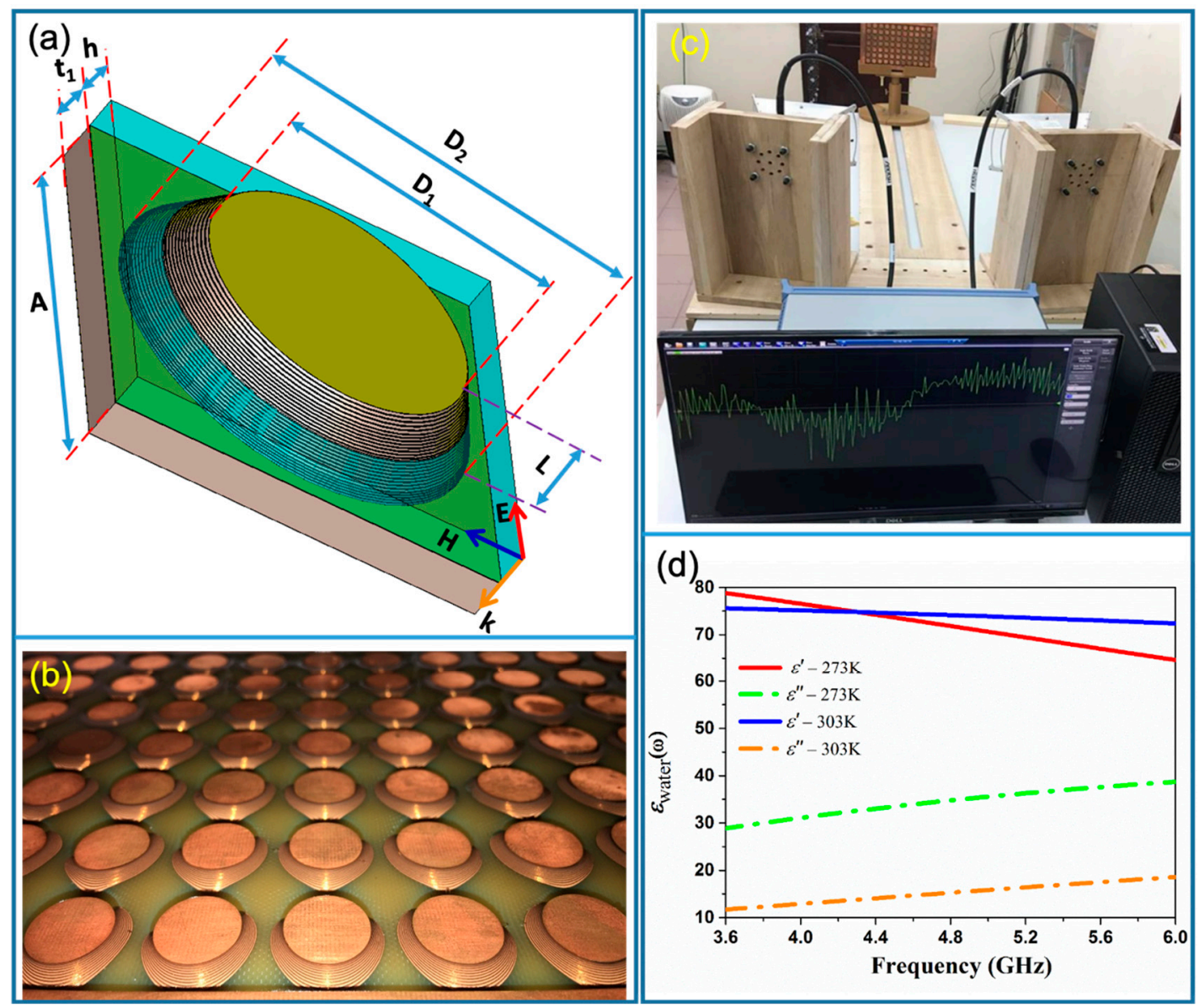

Figure 1. (a) Three-dimensional schematic of the water-hybrid metamaterial absorbers (MA); (b) fabricated truncated cone-type resonator (TCR)-MA hybridized with water; (c) measurement setup for the proposed TCR-MA by using ZNB20 vector network analyzer; (d) real part $\varepsilon^{\prime}$ and imaginary part $\varepsilon^{\prime \prime}$ of the complex permittivity for water at $273 \mathrm{~K}$ and $303 \mathrm{~K}$.

The precise milling and the heat pressing processes were simultaneously applied to fabricate a TCR as shown in Figure $1 \mathrm{~b}$. In order to integrate water, 10 by 12 grids of unit cells were kept inside a boundary wall, which has the same height as TCRs. The temperature of water was kept at $303 \mathrm{~K}$ (room temperature). The characteristics of switchable narrow/broad-band absorption were estimated by the reflection spectra measured with a ZNB-20 vector network analyzer (VNA) with a pair of radiating/detecting horn antennae, as shown in Figure 1c. The absorption (A) was calculated by $\mathrm{A}=1-|\mathrm{T}|-|\mathrm{R}|=1-\left|S_{21}(\omega)\right|^{2}-\left|S_{11}(\omega)\right|^{2}$, where $\mathrm{T}=\left|S_{21}(\omega)\right|^{2}$ and $\mathrm{R}=\left|S_{11}(\omega)\right|^{2}$ were the transmission and the reflection, respectively. In our structure, the thickness of bottom continuous copper layer was $36 \mu \mathrm{m}$, which was much thicker than the penetration depth of copper in the investigated frequency range (the penetration depth was calculated approximately to be $1 \mu \mathrm{m}$ at $5 \mathrm{GHz}$ ). Therefore, the transmission of bottom layer was minimized to be zero $\left[S_{21}(\omega)=0\right]$, since the EM wave could penetrate through the thickness of copper film at the frequencies of interest. Consequently, the absorption was basically calculated as $A=1-\left|S_{11}(\omega)\right|^{2}$, where $S_{11}(\omega)$ can be directly extracted from the CST Microwave Studio (for simulation) and measured by using ZNB-20 VNA (for experiment).

In the frequency range of interest, the permittivity of water was approximately defined by the Debye formula $[32,33]$ in CST Microwave Studio:

$$
\varepsilon(\omega)=\varepsilon_{\infty}(T)+\frac{\varepsilon_{S}(T)-\varepsilon_{\infty}(T)}{1-i \omega \tau(T)},
$$


and,

$$
\begin{gathered}
\varepsilon_{S}(T)=a_{1}-b_{1} T+c_{1} T^{2}-d_{1} T^{3}, \\
\varepsilon_{\infty}(T)=-a_{2} \exp \left(-b_{2} T\right)+\varepsilon_{S}(T), \\
\tau(T)=c_{2} \exp \left(T_{2} /\left(T+T_{1}\right)\right),
\end{gathered}
$$

where, $\varepsilon_{s}(T), \varepsilon_{\infty}(T)$ and $\tau(T)$ were the static permittivity, the high-frequency temperature dependent permittivity and the rotational relaxation time, respectively. In these Equations, $a_{1}=87.9, b_{1}=0.404 \mathrm{~K}^{-1}$, $c_{1}=9.59 \times 10^{-4} \mathrm{~K}^{-2}, d_{1}=1.33 \times 10^{-6} \mathrm{~K}^{-3}, a_{2}=80.7, b_{2}=4.42 \times 10^{-3} \mathrm{~K}^{-1}, c_{2}=1.37 \times 10^{-13} \mathrm{~s}, T_{1}=406 \mathrm{~K}$ and $T_{2}=924 \mathrm{~K}$. It is well recognized that the permittivity of water depends on the intrinsic temperature. The real and the imaginary parts of dielectric constant of water in the investigated $\mathrm{GHz}$ range are shown in Figure 1d, when the intrinsic temperature was changed from 303 to $273 \mathrm{~K}$. The imaginary part indicates a relatively low dielectric loss (or low conductivity of $1.59 \mathrm{~S} / \mathrm{m}$ ) because of the small value [28].

To estimate the dependence of effective impedance of TRC on the volume of water, the effective permittivity $\left[\varepsilon_{e f f}(\omega)\right]$ and the effective permeability $\left[\mu_{e f f}(\omega)\right]$ can be calculated by [34]

$$
\begin{aligned}
& \varepsilon_{\text {eff }}(\omega)=1+\frac{1}{d}\left[\frac{2 j\left(S_{11}(\omega)+1\right)}{k_{0}\left(S_{11}(\omega)-1\right)}\right], \\
& \mu_{\text {eff }}(\omega)=1+\frac{1}{d}\left[\frac{2 j\left(S_{11}(\omega)-1\right)}{k_{0}\left(S_{11}(\omega)+1\right)}\right] .
\end{aligned}
$$

Here, $d$ and $k_{0}$ are the distance to be traveled by the incident wave and the wave number of free space, respectively. Consequently, the total impedance between TRC and the surrounding environment $\left(\mathrm{Z}_{0}=\sqrt{\mu_{0} / \varepsilon_{0}}=377 \Omega\right.$ ) be effectively switched for the perfect matching in narrow/broad-band as

$$
Z=\sqrt{\mu / \varepsilon} \cdot \sqrt{\frac{\left(1+S_{11}(\omega)\right)^{2}-S_{21}^{2}(\omega)}{\left(1-S_{11}(\omega)\right)^{2}-S_{21}^{2}(\omega)}}=\sqrt{\frac{\left(1+S_{11}(\omega)\right)^{2}}{\left(1-S_{11}(\omega)\right)^{2}}}
$$

In other words, the specific frequency range allowing $\varepsilon_{e f f}(\omega)=\mu_{e f f}(\omega)$ [or $Z=Z_{0}$ ] was dominated by the height of water in the TRC. This was why, when the water was full between a pair of metallic plates of each sandwiched layer in TCR, the effective capacitor for equivalent LC circuit was reduced, owing to the conductive property of water. The efficiency of modulation for the proposed TRC was evaluated by the fractional bandwidth (FBW), where $f_{\text {low }}$ and $f_{\text {high }}$ indicate the lowest and the highest frequencies where absorption was over $90 \%$, respectively,

$$
F B W=\frac{2\left(f_{\text {high }}-f_{\text {low }}\right)}{f_{\text {high }}+f_{\text {low }}} .
$$

\section{Results}

Figure 2a shows the comparison between simulated and measured absorption spectra in case of no water $(h=0)$ and water integrated $(h=4.0 \mathrm{~mm})$ at $303 \mathrm{~K}$. Without water, the prediction by Equation (6) of a wideband absorption of FBW $=27.6 \%$ is in good agreement with both simulation and experiment. Obviously, Figure $2 \mathrm{~b}$ presents that, without water in the range where the absorption was over $90 \%$ (around 4.0 to $5.28 \mathrm{GHz}$ ), the real part of effective impedance tends toward one while the imaginary part was suppressed. Meanwhile, when $h=4.0 \mathrm{~mm}$ the wide-band absorption was switched to be narrow band with an absorption of $40 \%$ at $4.74 \mathrm{GHz}$.

The dependence of simulated broadband absorption on the height $(h)$ of water at different temperatures are shown in Figure 3. It was found that the increase of intrinsic temperature (from 273 to $303 \mathrm{~K}$ ) of water affected slightly the FBW of TCR absorber. The value of FBW gradually decreases $(27.6 \%, 21.6 \%, 18.2 \%, 15.6 \%$ and $7.4 \%)$ as $h$ was increased $(0,1.0,1.5,2.0$ and $2.5 \mathrm{~mm})$ in Figure $3 \mathrm{a}, \mathrm{b}$. At $h=3.5 \mathrm{~mm}$, the wide-band absorption was switched to be narrow band with absorption of $76 \%$ 
at $5.29 \mathrm{GHz}$ (for $273 \mathrm{~K}$ ) and $79 \%$ at $5.28 \mathrm{GHz}$ (for $303 \mathrm{~K}$ ). It was further predicted that, for a given $h$, the FBW was nearly constant as the temperature of water was varied from 273 to $303 \mathrm{~K}$. In addition, Figure $3 c, d(T=303 \mathrm{~K})$, shows that the real and the imaginary parts of $Z(\omega)$ tend to be nearly 1.0 and 0 , respectively as calculated by Equation (5), in the respective frequency range [4.25-5.28 GHz $(h=1.0 \mathrm{~mm}), 4.41-5.28 \mathrm{GHz}(h=1.5 \mathrm{~mm}), 4.57-5.26 \mathrm{GHz}(h=2.0 \mathrm{~mm})$ and $4.82-5.2 \mathrm{GHz}(h=2.5 \mathrm{~mm})]$ where absorption exceeds $90 \%$. It is well known that, in these frequency ranges, the real and the imaginary parts of effective permittivity and permeability were approximately equal $[\operatorname{Re}(\mu) \approx \operatorname{Re}(\varepsilon)$; $\operatorname{Im}(\mu) \approx \operatorname{Im}(\varepsilon)]$ in Equations (3) and (4). Furthermore, the imaginary part of impedance was always positive in the investigated frequency range. This condition implies that the dispersion of permeability was more important than that of permittivity [35]. In other words, the observation suggests that the magnetic resonances were dominant instead of the electric ones in the proposed MA.
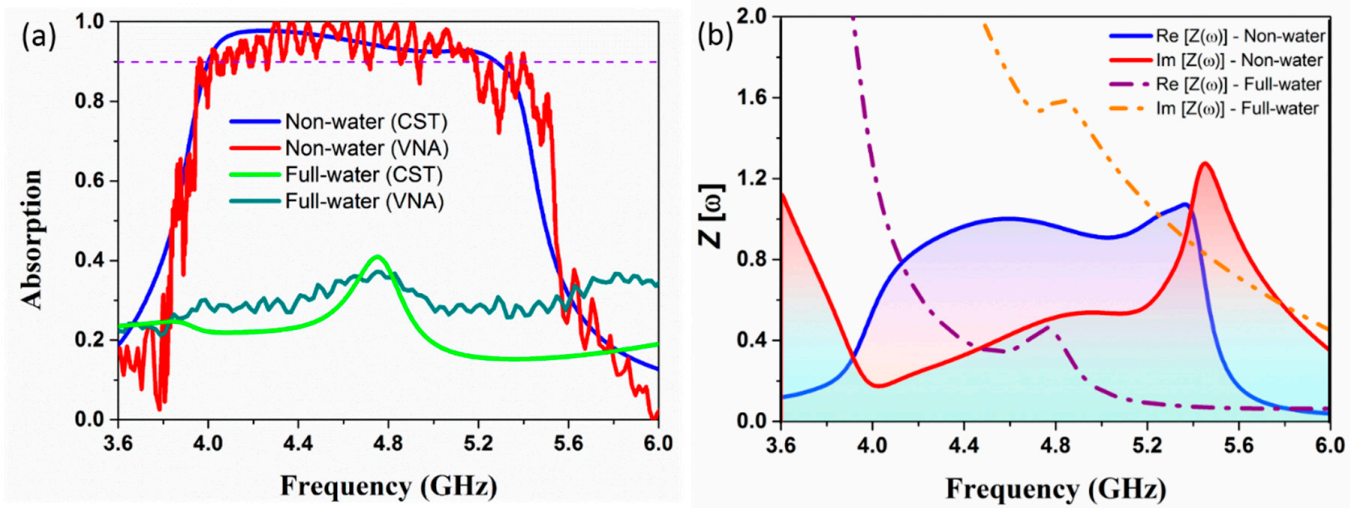

Figure 2. (a) Simulated (CST Microwave Studio) and measured (vector network analyzer (VNA)) absorption spectra in case of water integrated $(h=4.0 \mathrm{~mm})$ and no water $(h=0)$ at $303 \mathrm{~K}$. (b) Calculated effective impedance of TCR structure for these two cases.


Figure 3. Simulated absorption spectra with respect to the height of water at temperature of (a) $273 \mathrm{~K}$ and (b) $303 \mathrm{~K}$. Calculated effective values of impedance at $303 \mathrm{~K}$ for (c) real and (d) imaginary parts. All color ranges denote the corresponding absorption over $90 \%$. 
For the experimental confirmation, the measured absorptions over $90 \%$ with an FBW of $22.6 \%$ (from 4.19 to $5.26 \mathrm{GHz}$, in Figure $4 \mathrm{a}$ ) can be tuned to be FBW=16\% (from 4.46 to $5.24 \mathrm{GHz}$, in Figure 4b) by increasing $h$ from 1.0 to $2.0 \mathrm{~mm}$. In the case of $h=3.0 \mathrm{~mm}$, in Figure $4 \mathrm{c}$, the measured absorption was maintained at a maximum value of $82 \%$ from 5.02 to $5.28 \mathrm{GHz}$. These obtained results indicate a good agreement with the simulated data.

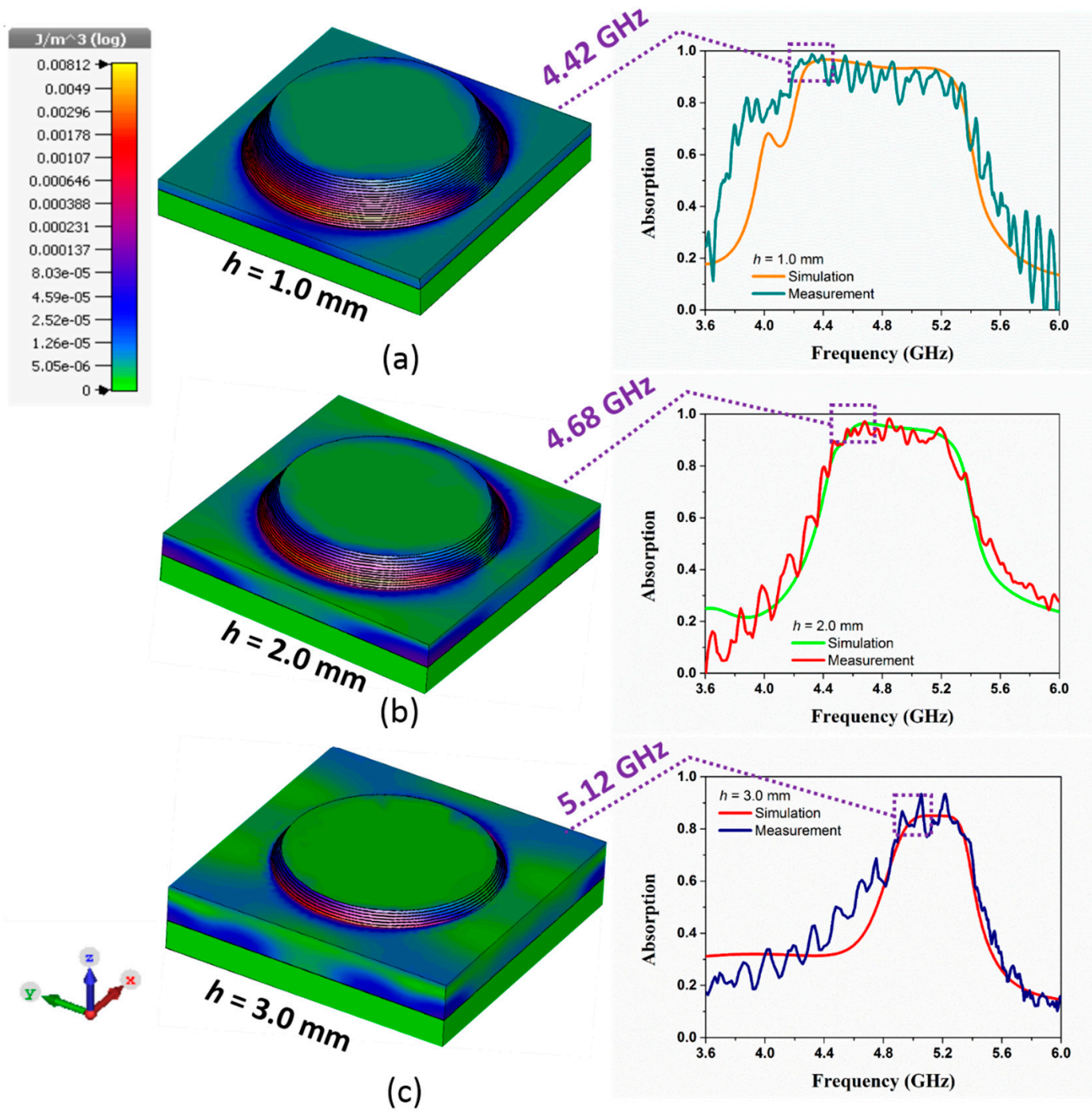

Figure 4. Distribution of the induced magnetic energy at the lowest absorption frequency and comparison between the simulated and measured absorption spectra at $303 \mathrm{~K}$ for $(\mathbf{a}) h=1.0,(\mathbf{b}) h=2.0$ and $(\mathbf{c}) h=3.0 \mathrm{~mm}$.

\section{Discussion}

It is normal that the underlying switching mechanism for narrow/broad-band absorption by utilizing a water-hybrid TCR relies on the distribution of induced magnetic energy as shown in Figure 4. As mentioned above, the broadband absorption feature of TRC structure was caused only by the magnetic resonances, which are induced by the antiparallel surface currents in successive layers (along the $k$ direction). As discussed deeply in our previous work on sandwiched-layer TCR [36], the induced electric and magnetic fields are excited continuously from the bottom to the top of TCR, leading to broad-band absorption. In other words, the varying diameters of these metallic disks result in close absorption peaks and water plays an important role in canceling or activating the anti-parallel surface currents flowing on them. Consequently, the modulation was triggered by 
the bottom-up cancellation of magnetic resonances (i.e., the low-frequency absorption peaks were gradually deactivated as the water-level rises). Thereafter, the induced magnetic energy was located and enhanced only in a specific volume of the TCR above the water level. Therefore, having both well-matched impedance and strong magnetic resonance at a given frequency was limited and depends on the injected-water level, as shown in Figure 3c,d. This impedes the ability of device to consume the energy of incoming EM waves at this switching frequency. The small deviations between measured and simulated absorption spectra in Figure 4 can be largely explained by the scattering from imperfections in the fabricated sample and the distorted shape of injected water due to its surface tension [37]. In spite of these discrepancies, our obtained results are robust enough for the stable modulation applications working in thermally variable conditions. The modulation performance of water-hybrid TRC structure was further predicted to be insensitive to the polarization angle of EM wave owing to its symmetric multilayered design (not shown here).

\section{Conclusions}

We proposed and demonstrated a simple model to control the bandwidth of near-perfect absorption of the TCR metamaterial by leveraging the hybridization with water. By exploiting the role of magnetic resonance in a multilayered structure, it was found that the relative-bandwidth of absorption spectrum, $\mathrm{FWB}=27.6 \%$ can be switched flexibly from $27.6 \%$ to $7.4 \%$ or absorption over $90 \%$ to below $80 \%$ in the WiMAX/LTE band. These results emphasize the important role of water in deactivating (or activating) induced magnetic resonances in the multilayered structure. This control method can provide further the insensitive operation to the polarization of incoming EM radiation and the thermal variations of injected water. The proposed model is an important step toward integrating MAs with the next generation of smart electronic components, especially, camouflage equipment, sensors and hygienic energy storage devices.

Author Contributions: B.X.K., V.T.H.H., Y.L., V.D.L. and Y.J.K. conceived the idea. Y.J.K. and B.X.K. fabricated the MA samples. B.X.K., V.T.H.H. and B.S.T. performed the measurements. The electromagnetic simulation and calculation were carried out by B.X.K., V.T.H.H., Y.L., H.-T.T. and L.Y.C. B.X.K., Y.L. and V.D.L. analyzed and wrote the article. All of the authors discussed and commented on the manuscript. All authors have read and agreed to the published version of the manuscript.

Funding: This research is funded by Graduate University of Science and Technology under Grant Number GUST.STS.ĐT2019-KHVL01.

Conflicts of Interest: The authors declare no conflict of interest.

\section{References}

1. Koschny, T.; Soukoulis, C.M.; Wegener, M. Metamaterials in microwaves, optics, mechanics, thermodynamics, and transport. J. Opt. 2017, 19, 084005. [CrossRef]

2. Landy, N.I.; Sajuyigbe, S.; Mock, J.J.; Smith, D.R.; Padilla, W.J. Perfect Metamaterial Absorber. Phys. Rev. Lett. 2008, 100, 207402. [CrossRef] [PubMed]

3. Knott, E.F.; Shaeffer, J.F.; Tuley, M.T. Radar Cross Section, 2nd ed.; SciTech Publishing, Inc.: NC, USA, 2004.

4. Paul, C.R. Introduction to Electromagnetic Compatibility; Wiley: Hoboken, NJ, USA, 2005.

5. Khuyen, B.X.; Tung, B.S.; Tung, N.T.; Hien, N.; Kim, Y.J.; Chen, L.-Y.; Lee, Y.P.; Linh, P.T.; Lam, V.D. Realization for dual-band high-order perfect absorption, based on metamaterial. J. Phys. D: Appl. Phys. 2019, 53, 105502. [CrossRef]

6. Tran, C.M.; Pham, H.V.; Nguyen, H.T.; Nguyen, T.T.; Vu, L.D.; Do, T.H. Creating Multiband and Broadband Metamaterial Absorber by Multiporous Square Layer Structure. Plasmonics 2019, 14, 1587-1592. [CrossRef]

7. Wu, P.; Chen, Z.; Jile, H.; Zhang, C.; Xu, D.; Lv, L. An infrared perfect absorber based on metal-dielectric-metal multi-layerfilms with nanocircle holes arrays. Results Phys. 2020, 16, 1029522. [CrossRef]

8. Park, H.; Lee, S.-Y.; Kim, J. Near-infrared coherent perfect absorption in plasmonic metal-insulator-metal waveguide. Opt. Express 2015, 23, 24464. [CrossRef] 
9. Li, W.; Guler, U.; Kinsey, N.; Naik, G.V.; Boltasseva, A.; Guan, J.; Shalaev, V.M.; Kildishev, A.V. Refractory Plasmonics with Titanium Nitride: Broadband Metamaterial Absorber. Adv. Mater. 2014, 26, 7959-7965. [CrossRef]

10. Hedayati, M.K.; Javaherirahim, M.; Mozooni, B.; Abdelaziz, R.; Tavassolizadeh, A.; Chakravadhanula, V.S.K.; Zaporojtchenko, V.; Strunkus, T.; Faupel, F.; Elbahri, M.; et al. Design of a Perfect Black Absorber at Visible Frequencies Using Plasmonic Metamaterials. Adv. Mater. 2011, 23, 5410-5414. [CrossRef]

11. Ding, F.; Cui, Y.; Ge, X.; Jin, Y.; He, S. Ultra-broadband microwave metamaterial absorber. Appl. Phys. Lett. 2012, 100, 103506. [CrossRef]

12. Yu, P.; Besteiro, L.V.; Huang, Y.; Wu, J.; Fu, L.; Tan, H.H.; Jagadish, C.; Wiederrecht, G.P.; Govorov, A.O.; Wang, Z. Broadband Metamaterial Absorbers. Adv. Opt. Mater. 2018, 7, 1800995. [CrossRef]

13. Deng, T.; Li, Z.-W.; Chua, M.-J.; Chen, Z.N. Broadband and Ultrathin Frequency-Dispersive Metamaterial Screen for Reflectivity Reduction. IEEE Trans. Antennas Propag. 2015, 63, 4156. [CrossRef]

14. Zhu, J.; Ma, Z.; Sun, W.; Ding, F.; He, Q.; Zhou, L.; Ma, Y. Ultra-broadband terahertz metamaterial absorber. Appl. Phys. Lett. 2014, 105, 021102. [CrossRef]

15. Yin, S.; Zhu, J.; Xu, W.; Jiang, W.; Yuan, J.; Yin, G.; Xie, L.; Ying, Y.; Ma, Y. High-performance terahertz wave absorbers made of silicon-based metamaterials. Appl. Phys. Lett. 2015, 107, 073903. [CrossRef]

16. Cong, L.L.; Cao, X.Y.; Song, T.; Gao, J.; Lan, J.X. Angular- and Polarization-insensitive Ultrathin Double-layered Metamaterial Absorber for Ultra-wideband Application. Sci. Rep. 2018, 8, 9627. [CrossRef]

17. Liu, H.; Wang, Z.-H.; Li, L.; Fan, Y.-X.; Tao, Z.-Y. Vanadium Dioxide-assisted Broadband Tunable Terahertz Metamaterial Absorber. Sci. Rep. 2019, 9, 5751. [CrossRef]

18. Chen, L.; Sun, L.; Dong, H.; Mou, N.; Zhang, Y.; Li, Q.; Jiang, X.; Zhang, L. Near-field imaging of the multi-resonant mode induced broadband tunable metamaterial absorber. RSC Adv. 2020, 10, 5146-5151. [CrossRef]

19. Zhang, H.; Ling, F.; Wang, H.; Zhang, Y.; Zhang, B. A water hybrid graphene metamaterial absorber with broadband absorption. Opt. Commun. 2020, 463, 125394. [CrossRef]

20. Huynh, T.V.; Tung, B.S.; Khuyen, B.X.; Tung, N.T.; Lam, V.D. Electrically tunable graphene-based metamaterials: A brief review. Mod. Phys. Lett. B 2019, 33, 1950404. [CrossRef]

21. Buchner, R.; Barthel, J.; Stauber, J. The dielectric relaxation of water between $0^{\circ} \mathrm{C}$ and $35^{\circ} \mathrm{C}$. Chem. Phys. Lett. 1999, 306, 57-63. [CrossRef]

22. Andryieuski, A.; Kuznetsova, S.M.; Zhukovsky, S.V.; Kivshar, Y.S.; Lavrinenko, A.V. Water: Promising Opportunities for Tunable All-dielectric Electromagnetic Metamaterials. Sci. Rep. 2015, 5, 13535. [CrossRef]

23. Shen, Y.; Zhang, J.; Pang, Y.; Zheng, L.; Wang, J.; Ma, H.; Qu, S. Thermally Tunable Ultra-wideband Metamaterial Absorbers based on Three-dimensional Water-substrate Construction. Sci. Rep. 2018, 8, 4423. [CrossRef] [PubMed]

24. Pang, Y.; Wang, J.; Cheng, Q.; Xia, S.; Zhou, X.-Y.; Xu, Z.; Cui, T.J.; Qu, S. Thermally tunable water-substrate broadband metamaterial absorbers. Appl. Phys. Lett. 2017, 110, 104103. [CrossRef]

25. Wu, Z.; Chen, X.; Zhang, Z.; Heng, L.; Wang, S.; Zou, Y. Design and optimization of a flexible water-based microwave absorbing metamaterial. Appl. Phys. Express 2019, 12, 057003. [CrossRef]

26. Zhou, Y.; Shen, Z.; Huang, X.; Wu, J.; Li, Y.; Huang, S.; Yang, H. Ultra-wideband water-based metamaterial absorber with temperature insensitivity. Phys. Lett. A 2019, 383, 2739-2743. [CrossRef]

27. Zhou, Y.; Shen, Z.; Wu, J.; Zhang, Y.; Huang, S.; Yang, H. Design of ultra-wideband and near-unity absorption water-based metamaterial absorber. Appl. Phys. A 2020, 126, 1-5. [CrossRef]

28. Yoo, Y.J.; Ju, S.; Park, S.Y.; Kim, Y.J.; Bong, J.; Lim, T.; Kim, K.W.; Rhee, J.Y.; Lee, Y.P. Metamaterial Absorber for Electromagnetic Waves in Periodic Water Droplets. Sci. Rep. 2015, 5, 14018. [CrossRef]

29. Song, Q.; Zhang, W.; Wu, P.C.; Zhu, W.; Shen, Z.X.; Chong, P.H.; Liang, Q.X.; Yang, Z.C.; Hao, Y.L.; Cai, H.; et al. Water-Resonator-Based Metasurface: An Ultrabroadband and Near-Unity Absorption. Adv. Opt. Mater. 2017, 5, 1601103. [CrossRef]

30. Pang, Y.; Shen, Y.; Li, Y.; Wang, J.; Xu, Z.; Qu, S. Water-based metamaterial absorbers for optical transparency and broadband microwave absorption. J. Appl. Phys. 2018, 123, 155106. [CrossRef]

31. Rybin, M.; Samusev, K.B.; Kapitanova, P.; Filonov, D.S.; Belov, P.A.; Kivshar, Y.S.; Limonov, M. Switchable invisibility of dielectric resonators. Phys. Rev. B 2017, 95, 165119. [CrossRef]

32. Ellison, W. Permittivity of pure water, at standard atmospheric pressure, over the frequency range $0-25 \mathrm{THz}$ and the temperature range 0-100॰C. J. Phys. Chem. Ref. Data 2007, 36, 1-18. [CrossRef] 
33. Liebe, H.J.; Hufford, G.A.; Manabe, T. A model for the complex permittivity of water at frequencies below 1 THz. Int. J. Infrared Millim. Waves 1991, 12, 659-675. [CrossRef]

34. Bhattacharyya, S.; Srivastava, K.V. Triple band polarization-independent ultra-thin metamaterial absorber using electric field-driven LC resonator. J. Appl. Phys. 2014, 115, 64508. [CrossRef]

35. Landau, L.D.; Lifshitz, E.M.; Pitaevskii, L.P. Electrodynamics of Continuous Media, 2nd ed.; Section 87, 301; Pergamon Press: New York, NY, USA, 1984.

36. Kim, Y.J.; Yoo, Y.J.; Kim, K.W.; Rhee, J.Y.; Kim, Y.H.; Lee, Y.P. Dual broadband metamaterial absorber. Opt. Express 2015, 23, 3861-3868. [CrossRef] [PubMed]

37. Zhao, J.; Wei, S.; Wang, C.; Chen, K.; Zhu, B.; Jiang, T.; Feng, Y.-J. Broadband microwave absorption utilizing water-based metamaterial structures. Opt. Express 2018, 26, 8522-8531. [CrossRef]

(C) 2020 by the authors. Licensee MDPI, Basel, Switzerland. This article is an open access article distributed under the terms and conditions of the Creative Commons Attribution (CC BY) license (http://creativecommons.org/licenses/by/4.0/). 